Cartas científicas

Rev Urug Cardiol 2021; 36: e36108

doi: 10.29277/cardio.36.1.8

\title{
Linfoma pericárdico primario a células T/NK. Reporte de un caso
}

\section{Resumen}

Los linfomas cardíacos primarios son un subtipo muy poco frecuente de tumor en los cuales la lesión primaria se encuentra en el corazón. Los tumores suelen ser infiltrantes y se localizan en la aurícula derecha, seguidos del pericardio. Su mortalidad es notablemente alta y el diagnóstico tardío es el principal factor para su mal pronóstico. Describimos el caso de un paciente que presentó shock obstructivo por derrame pericárdico profuso causado por un tipo raro de tumor cardíaco primario, un linfoma pericárdico de células T/NK.

Palabras clave: $\quad$ TUMORES CARDÍACOS

TUMORES PERICÁRDICOS

DERRAME PERICÁRDICO

TAPONAMIENTO CARDÍACO

Primary pericardial T/NK cell lymphoma. Case report

\section{Summary}

Primary cardiac lymphomas are a rare subtype of lymphomas in which the primary lesion is in the heart. The tumors are usually located in the right atria, followed by the pericardium and are frequently infiltrative. Mortality is remarkably high in this group and the delayed diagnosis is the main factor for its poor prognosis. We describe the case of a patient that presented with obstructive shock due to profuse pericardial effuse caused by a rare kind of primary cardiac tumor, a T/NK cell pericardial lymphoma.

Key words: $\quad$ CARDIAC TUMORS

PERICARDIAL TUMORS

PERICARDIAL EFFUSION

PERICARDIAL TAMPONADE

Linfoma pericárdico primário de células T/NK. Relato de um caso

\section{Resumo}

Os linfomas cardíacos primários são um subtipo de tumor muito raro, no qual a lesão primária está no coração. Os tumores geralmente são infiltrativos e localizam-se no átrio direito, seguidos pelo pericárdio. Sua mortalidade é notavelmente alta e o diagnóstico tardio é o principal fator que produz seu mau prognóstico. Descrevemos o caso de um paciente que apresentou choque obstrutivo devido a um derrame pericárdico profuso causado por um tipo raro de tumor cardíaco primário, um linfoma pericárdico de células T/NK.

Palavras chave: TUMORES CARDÍACOS

TUMORES PERICÁRDICOS

DERRAME PERICÁRDICO

TAMPONAMENTO CARDÍACO

\section{Sr. Editor:}

\section{Introducción}

Los tumores cardíacos pueden clasificarse en primarios o secundarios (metastásicos). Los tumores primarios son extremadamente infrecuentes, con una incidencia estimada de $0,05 \%, 40$ veces menos frecuentes que los tumores cardíacos secundarios.
De ese pequeño número, el 10\% corresponde a tumores malignos: $95 \%$ a sarcomas cardíacos y el $5 \%$ restante a linfomas o mesoteliomas.

\section{Presentación del caso}

Mujer de 74 años con antecedentes personales de hipotiroidismo, enfermedad celíaca y portadora de fibrilación auricular paroxística. Un año previo a la

Recibido Ene 7, 2021; aceptado Ene 15, 2021. 


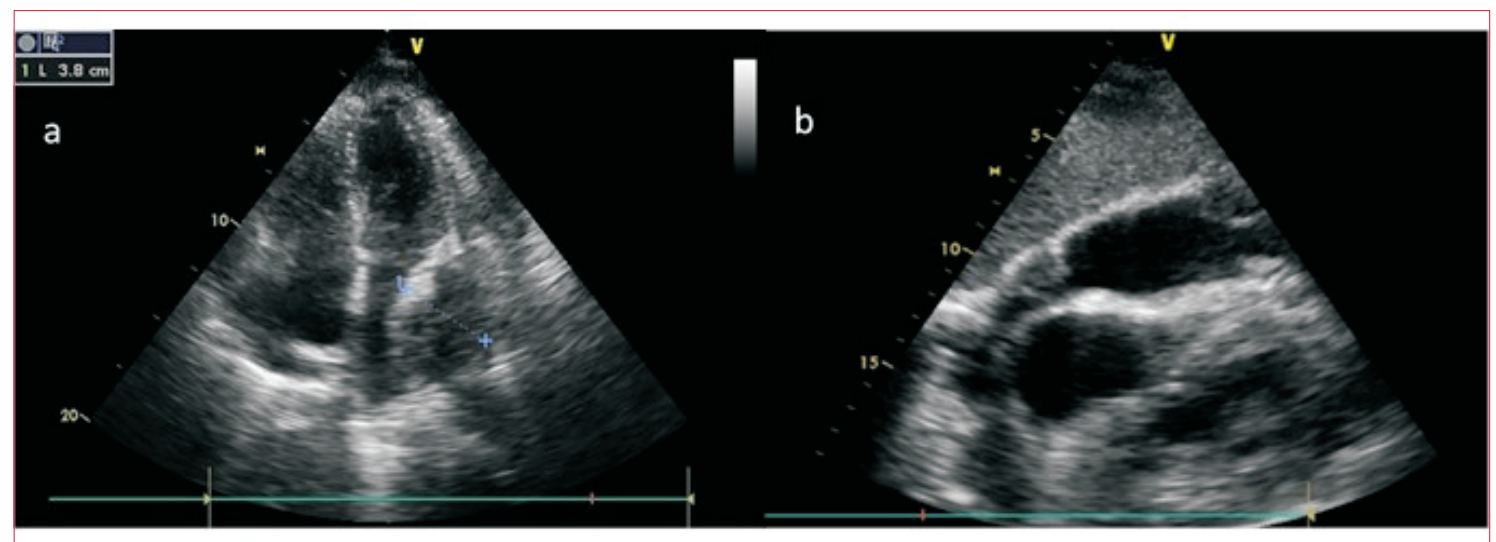

Figura 1. a) Ecocardiograma transtorácico vista apical cuatro cámaras: se observa gran masa pericárdica comprimiendo la aurícula izquierda. b) Vista subcostal que muestra un derrame pericárdico severo y múltiples masas nodulares localizadas a nivel pericárdico.

consulta se diagnosticó un tumor pancreático benigno, extirpado de forma completa, sin necesidad de tratamiento posterior. $\mathrm{Al}$ momento del contacto médico recibía tratamiento con $\mathrm{T} 4$ y calcioantagonistas. No presentaba antecedentes familiares cardiovasculares de relevancia.

La paciente fue admitida en el departamento de emergencia por disnea de esfuerzo progresiva que se hizo de reposo en el momento de la consulta, y dolor precordial de seis días de evolución. Al examen físico, se destacaba polipnea de 26 respiraciones por minuto, frecuencia cardíaca de 70 ciclos por minuto, presión arterial de 100/60 mmHg, palidez cutáneo-mucosa, sin signos de hipoperfusión periférica, sin fiebre. Saturación de oxígeno ventilando aire ambiente de $95 \%$. A la auscultación ruidos cardíacos hipofonéticos, sin soplos. Ingurgitación yugular, reflujo hepatoyugular y edemas gravitacionales en ambos miembros inferiores hasta las rodillas. Pulsos arteriales periféricos presentes y simétricos. Estertores crepitantes en extrema base de ambos campos pulmonares.

El electrocardiograma del ingreso reveló un ritmo sinusal con onda $\mathrm{P}$ e intervalo PR normales, eje eléctrico medio normoposicionado a $0^{\circ}$, microvoltaje de los complejos QRS y alteraciones difusas de la repolarización dadas por onda T invertida, asimétrica y aplanada en las derivaciones precordiales.

La radiografía de tórax mostró un mediastino ensanchado en forma de "botellón", sugerente de derrame pericárdico severo. Escaso edema intersticio-alveolar difuso y borramiento de ambos ángulos costofrénicos.

Se decidió realizar un ecocardiograma transtorácico (ETT), el cual confirmó la presencia de un derrame pericárdico severo, a predominio posterior, y una gran masa intrapericárdica de 80 por $38 \mathrm{~mm}$ aproximadamente, con múltiples imágenes nodulares a nivel del pericardio parietal. Ventrículo iz- quierdo de dimensiones normales, con fracción de eyección preservada. Vena cava inferior francamente dilatada, con colapso inspiratorio disminuido, sugestivo de presiones auriculares derechas elevadas. También se observó un derrame pleural izquierdo significativo con atelectasia pasiva a dicho nivel (figura 1).

Se realizó tomografía computarizada de tórax que confirmó la presencia de una gran masa sólida y heterogénea a nivel de la parte central del pericardio posterior de 67 por 82 por $54 \mathrm{~mm}$, con signos de compresión de la aurícula izquierda y de las venas pulmonares, así como múltiples masas nodulares localizadas a nivel del pericardio parietal (figura 2).

En la evolución inmediata la paciente presentó deterioro hemodinámico significativo, con progresión a shock obstructivo. Se realizó drenaje pericárdico de emergencia, con extracción de 400 cc de fluido hemático, con inmediata mejora de los parámetros hemodinámicos.

Posteriormente se trasladó a block quirúrgico, donde se realizó biopsia de la masa pericárdica y se dejó colocado un drenaje pericárdico, con gasto mantenido de líquido hemático.

La inspección macroscópica de la masa reveló que se trataba de un tumor maligno, con alta probabilidad de que correspondiera a un linfoma. La tomografía de emisión de positrones (PET-TC) mostró una extensa masa hipermetabólica, localizada en la porción posterior del pericardio, con compresión de las cavidades cardíacas, principalmente de la aurícula izquierda, pero sin infiltrado miocárdico. A nivel anterior, en proximidad al diafragma, presentaba otra masa de similares características, a nivel pericárdico, de 22 mm. No se observó compromiso de médula ósea (figura 3). 


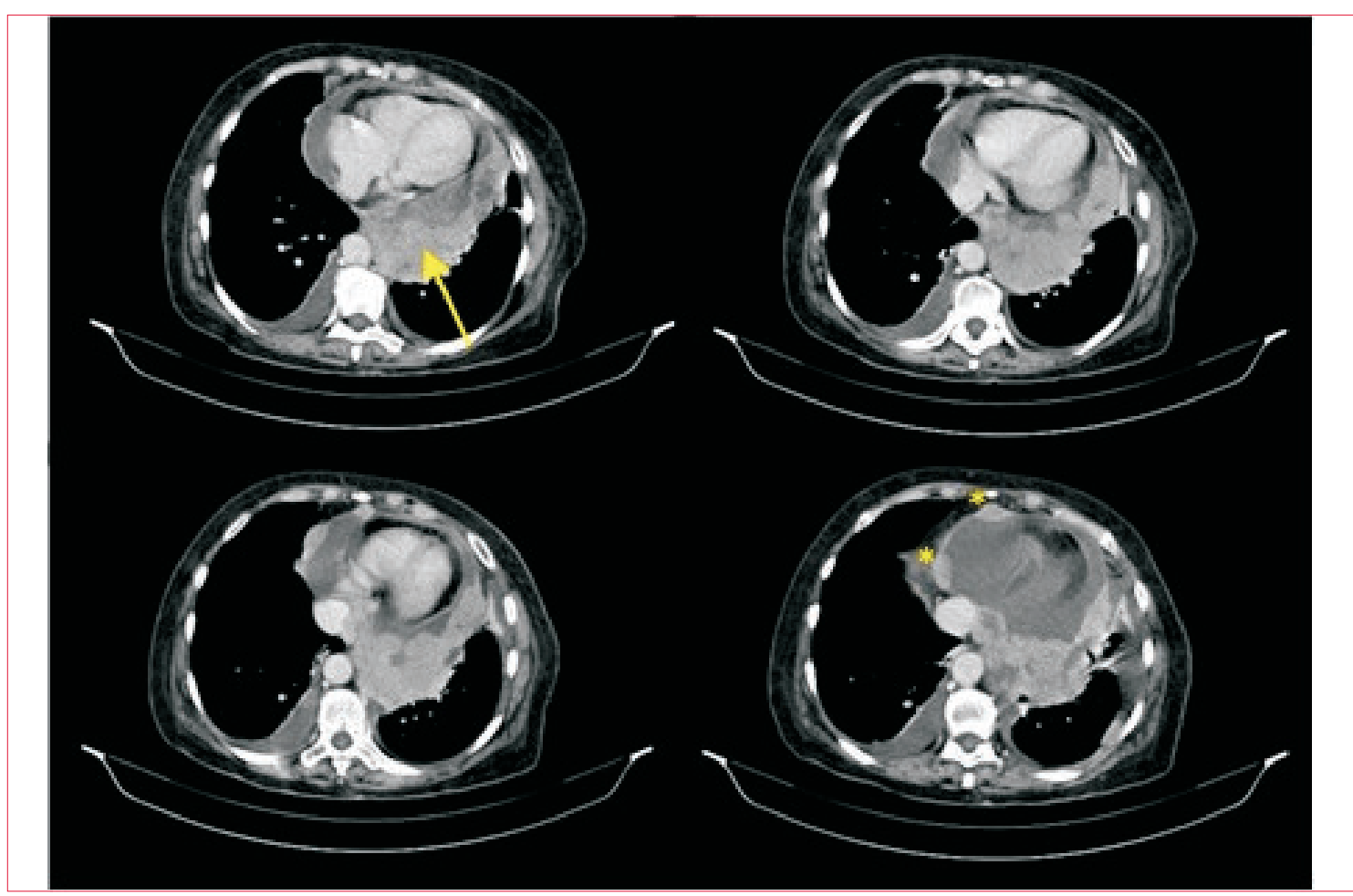

Figura 2. Tomografía computarizada de tórax. Se constata gran masa pericárdica heterogénea y sólida a nivel posterior (flecha) y múltiples masas nodulares localizadas en el pericardio(*).

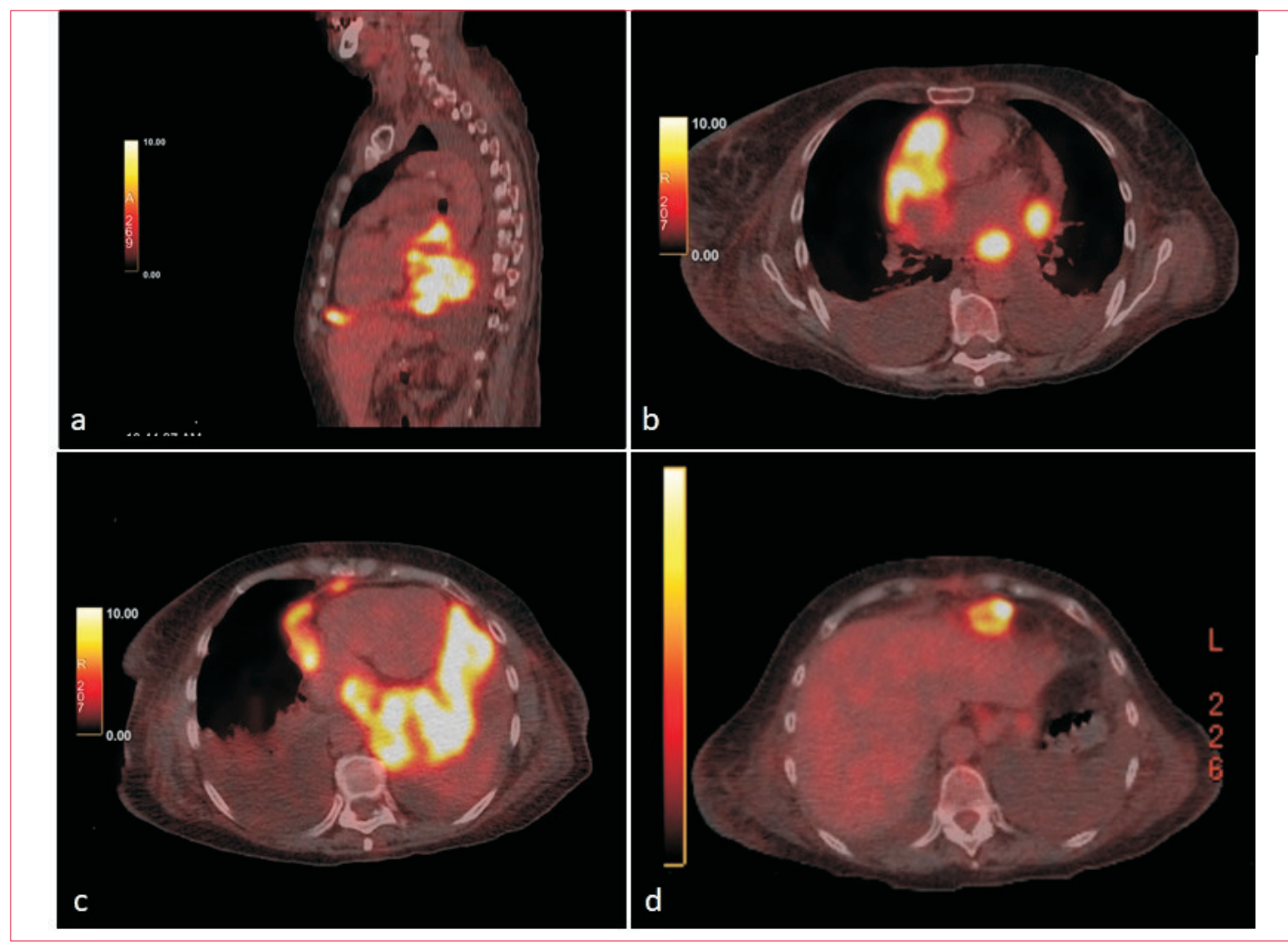

Figura 3. PET-TC. a) Masa hipermetabólica sólida en el pericardio posterior y agrandamientos nodulares en el pericardio anterior en relación con el diafragma. b) y c) Extensión de la masa hacia la parte lateral del pericardio, pero sin infiltración miocárdica. d) Masa nodular anterior. 

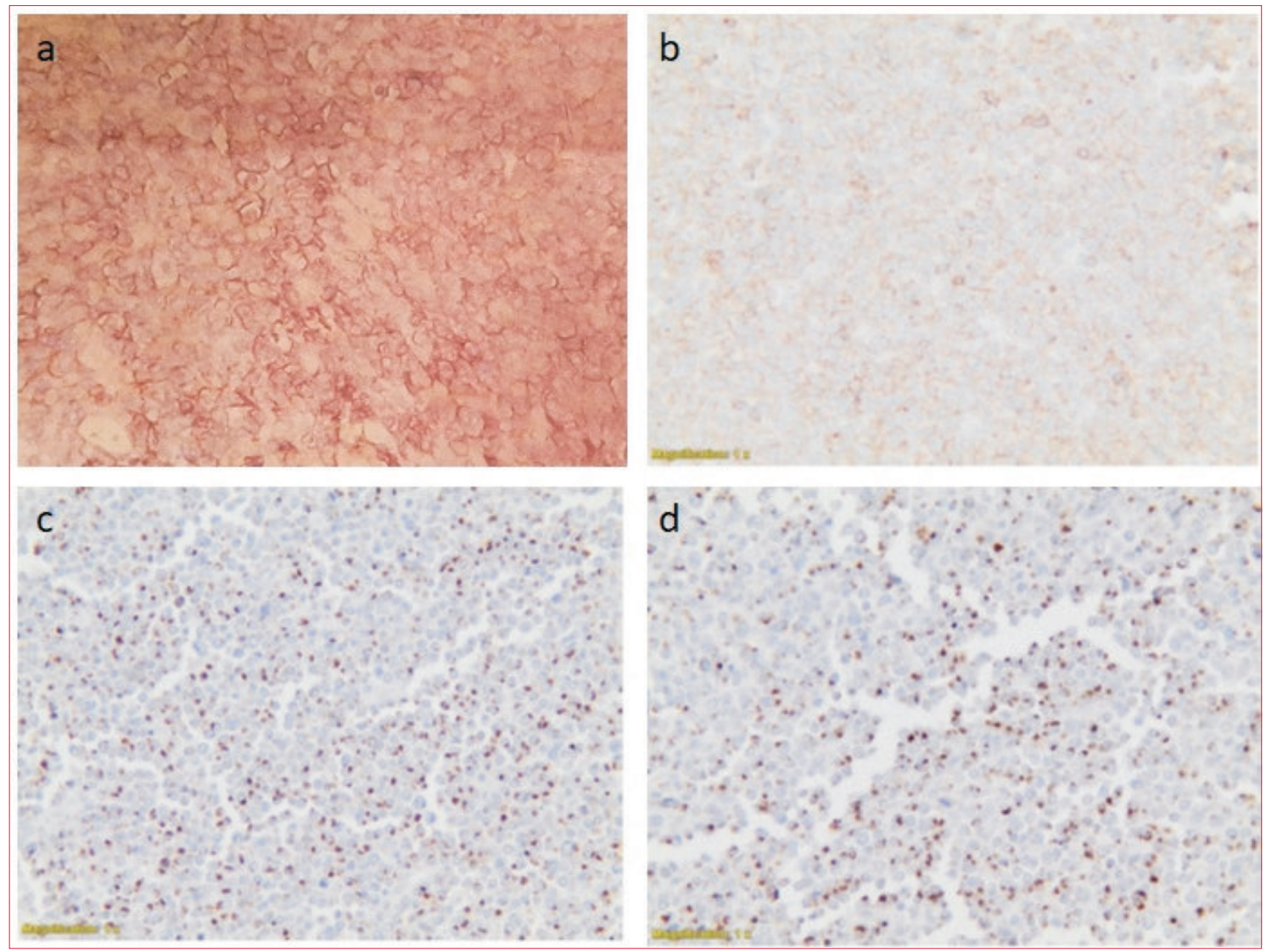

Figura 4. Biopsia pericárdica y análisis inmuno-histoquímico. a) Intenso marcado ACL (CD45RO), >90\%. b) Intenso marcado CD8+. c) Marcado focal de CD56 con intensa expresión de gránulos citotóxicos de granzima B. d) Marcado TIA-1. Compatible con linfoma extranodal tipo nasal a células T/NK.

El análisis histopatológico de la masa la identificó como un linfoma no Hodgkin extranodal a células T/NK (natural killer) de tipo nasal (figura $4)$.

La paciente inició tratamiento quimioterápico, con rápida evolución a la peoría, falleciendo a las dos semanas del ingreso hospitalario.

\section{Discusión}

Los linfomas cardíacos primarios son un subtipo de linfomas extremadamente raros, en los que la lesión primaria se localiza a nivel del corazón o del pericardio $^{(1)}$. Generalmente son de células B y ocurren predominantemente en pacientes inmunosuprimidos o inmunodeprimidos. En pacientes sin enfermedades inmunes son muy raros y representan aproximadamente el 1,3\% de todos los tumores cardíacos prima$\operatorname{rios}^{(2)}$. Por otro lado, los linfomas cardíacos secundarios, lo que corresponde a linfomas extracardíacos con compromiso miocárdico, son más frecuentes, con una incidencia del $20 \%$ de todos los linfomas ${ }^{(3)}$.

La localización más frecuente de los linfomas cardíacos primarios es la aurícula derecha, seguida del pericardio ${ }^{(4)}$. Estos pacientes generalmente de- sarrollan síntomas cardiovasculares inespecíficos, dependiendo principalmente de la localización del tumor. Las manifestaciones más frecuentes son las arritmias, el síncope, la miocardiopatía restrictiva, el dolor torácico y la disnea.

En el ETT, estos tumores son vistos usualmente como una masa homogénea, infiltrante, que produce engrosamiento pericárdico y fisiología constrictiva. También pueden verse como masas nodulares que invaden las cavidades cardíacas, con tendencia a infiltrar la aurícula o el ventrículo derecho, o ambos. Otras imágenes que pueden ser de gran utilidad son la tomografía computada y la resonancia cardíaca. El PET-CT con 18-FDG también puede revelar información crucial para estratificar la enfermedad, planear el tratamiento y evaluar la respuesta(5).

Independientemente de la gran utilidad de todas las técnicas de imagen nombradas, el diagnóstico definitivo se realiza únicamente con estudio histopatológico de la masa en cuestión. Estas técnicas incluyen análisis tanto del líquido pericárdico como de la masa cardíaca o pericárdica.

Con respecto al tratamiento, la quimioterapia es la mejor opción para estos tumores. Sin embargo, el pronóstico de estos pacientes es extremadamente 
malo, siendo el diagnóstico tardío el principal factor que marca el pronóstico a corto y largo plazo. Más del $60 \%$ de estos pacientes muere dentro de los primeros dos meses de hecho el diagnóstico, a pesar de tratamiento óptimo ${ }^{(6)}$.

\section{Conclusión}

Los tumores cardíacos primarios son una entidad extremadamente infrecuente y rara vez son de etio- logía maligna. Cuando la malignidad del tumor se confirma, la mayoría de éstos corresponden a sarcomas, seguidos a distancia de los linfomas y mesoteliomas. Los linfomas cardíacos primarios usualmente afectan las cámaras cardíacas derechas o el pericardio, o ambos. Son frecuentemente infiltrantes y el diagnóstico tardío es el factor que sella el pronóstico sombrío de estos pacientes, en los que, a pesar del tratamiento médico óptimo, la mortalidad permanece muy alta.

\section{Guillermo Agorrody ${ }^{1,2}$, Amparo Fernández ${ }^{1,2}$, Facundo Ríos ${ }^{1,2}$, María José Arocena ${ }^{1,2}$, Lilián Díaz ${ }^{3}$, Jorge Estigarribia ${ }^{1}$ \\ 1. Instituto Nacional de Cirugía Cardíaca (INCC). Montevideo, Uruguay. 2. Departamento de Cardiología, Servicio Médico Integral (SMI). Montevideo, Uruguay.}

Guillermo Agorrody, https://orcid.org/0000-0002-9241-6681

Amparo Fernández, https://orcid.org/0000-0003-4243-7844

Facundo Ríos, https://orcid.org/0000-0002-6868-3480

María José Arocena, https://orcid.org/0000-0003-4072-7426

Lilián Díaz, https://orcid.org/0000-0002-1056-2379

Jorge Estigarribia, https://orcid.org/0000-0002-5136-7668

Este artículo fue aceptado para su publicación por la Editora jefa, Dra. María Victoria Ramos.

3. Departamento de Hematología, Servicio Médico Integral (SMI). Montevideo, Uruguay. Correspondencia: Dr. Guillermo Agorrody.Correo electrónico: guillermo.agorrody@gmail.com

\section{Bibliografía}

1. Carras S, Berger F, Chalabreysse L, Callet-Bauchut E, Cordier J-F, Salles G, et al. Primary cardiac lymphoma: diagnosis, treatment and outcome in a modern series. Hematol Oncol. 2017; 35(4):510-9. doi: 10.1002/hon.2301.

2. Tyebally S, Chen D, Bhattacharyya S, Mughrabi A, Hussain Z, Manisty C, et al. Cardiac Tumors: JACC Cardio oncology state-of-the-art review. JACC: CardioOncology. 2020;2(2):293-311. doi.10 1016/j.jaccao.2020.05.009

3. Gowda RM, Khan IA. Clinical perspectives of primary cardiac lymphoma. Angiology. 2003;54(5):599-604. doi: 10.1177/000331970305400510.
4. Flox Camacho Á, Hernández Hernández F, Salguero Bodes R, Sánchez Pérez I, Carbonell Porras A, Tascón Pérez J. Linfoma cardíaco primario: diagnóstico mediante biopsia transyugular. Rev Esp Cardiol. 2003;56(11):1141-4. doi: 10.1157/13054042

5. Bianchi Castelli J, Alexandre L, Futuro G, Scanavacca M, Soares Júnior J. Primary cardiac lymphoma detected by $18 \mathrm{~F}-F D G$ PET scan: a case report. J Nucl Cardiol. 2011;18(5):974-7. doi: 10.1007/ s12350-011-9418-4.

6. Lamba G, Frishman WH. Cardiac and pericardial tumors. Cardiol Rev. 2012;20(5):237-52. doi: 10. 1097/CRD.0b013e31825603e7 